\title{
Daz slüzzelîn
}

\section{Karl Rihaczek}

\section{Liebe Leserinnen, liebe Leser!}

Sie kennen das Verslein „Dû bist mîn, ich bin dîn...". Darin sagt eine Dame, ihr Geliebter sei in ihrem Herzen eingeschlossen und müsse für immer drin bleiben, weil das Schlüsselein verloren sei. ${ }^{1}$ Damit sagt sie, dass dieser ihr Herzenszustand endgültig und unveränderlich, versiegelt sei. Der Gedanke an den Schlüsselverlust gibt ihr offensichtlich ein Glücksgefühl. Ein seltener Fall. Denn üblicherweise fürchtet der Mensch einen Schlüsselverlust.

Zum Beispiel: Wir waren mit Harry auf einer längeren Autoreise unterwegs und lebten zum Teil aus dem Kofferraum. Immer wenn Harry sich im Kofferraum umgetan hatte und im Begriff war, ihn zuzuklappen, hielt er inne, zeigte mir den Schlüssel und sagte: „Karl, I am very superstitious. Key in trunk means bad luck!" AVIS hatte uns nur einen einzigen Schlüssel mitgegeben. Da kann es der Teufel wollen, dass irgendwann der Kofferraum zuschnappt und der Schlüssel darinnen eingeschlossen ist. Man will zum Beispiel einen Koffer herausholen, braucht dazu beide Hände, legt den Schlüssel hin, vergisst ihn dort für eine Sekunde, klappt den Kofferraumdeckel zu, will weiterfahren und steht dumm da. Verlorn ist daz slüzzelîn. Und je besser so ein verlorener Schlüssel sichert, umso verlorener ist er. Man ist damit am Ende. Es hilft nur mehr irreguläre kostspielige Gewalt. Harrys „Aberglauben“ war also rational gut unterlegt. Der verlorene, nicht mehr verfügbare Schlüssel ist ein Unglück. Harry und das Verslein vermitteln also gegensätzliche Erkenntnisse zum gleichen Thema.

Und nun zum asymmetrischen RSAAlgorithmus. Der sieht bekanntlich zwei komplementäre Schlüssel vor, einen öffentlichen und einen geheimen. Mit dem geheimen kann man Digitales unterschreiben, mit dem öffentlichen kann man die Unterschrift verifizieren. Den geheimen gibt es

\footnotetext{
'Dû bist mîn, ich bin dîn:

des solt dû gewis sîn;

dû bist beslozzen in mînem herzen,

verlorn ist daz slüzzelîn:

dû muost och immer darinne sîn.
}

nur in einer einzigen Ausfertigung. Wenn man ihn verliert, kann man - logisch - mit ihm nicht mehr unterschreiben. Man kann zwar den Klartext verändern; dann stimmt aber die Unterschrift nicht. Die Manipulation lässt sich ohne Schlüssel nicht unkenntlich machen. So Unterschriebenes kann also nicht mehr gefälscht werden. Schlüsselverlust ein Glücksfall?

Stellen wir uns vor: Shakespeare hätte für sich ein RSA-Schlüsselpaar generiert, seine dichterische Produktion mit dem Unterschriftsschlüssel unterschrieben und danach diesen Schlüssel in Meerestiefen versenkt. Dann ließe sich (mit dem öffentlichen Schlüssel) die Echtheit der Schriften jederzeit eindeutig nachweisen, denn jede später eingebrachte Veränderung würde bei der Verifikation der Unterschrift sofort auffallen. Man könnte meinen: Die mannigfachen Zweifel der Literaturwissenschaftler an der Originalität der überlieferten Shakespeare'schen Werke wären gar nicht aufgekommen, könnte doch das per Unterschrift Versiegelte unmöglich manipuliert worden sein. Ein vernichteter, unauffindbarer Schlüssel bedeutete also in diesem Falle einen Sicherheitsgewinn - means good luck. Wie im Falle der Dame mit dem verlorenen Schlüsselein vermittelt seine Unauffindbarkeit ein positives Gefühl. Das ist freilich wie dieses ebenso ungewöhnlich, denn in der Regel bringt, wie gesagt, der Verlust eines Schlüssels - siehe Schlüssel im zugeklappten Kofferraum - Ärger und Unsicherheit. Ärger, weil man ihn braucht und ihn nicht zur Hand hat; Unsicherheit, weil er vom Falschen aufgefunden und missbraucht werden kann. Beeindruckend, dass es sich mit dem asymmetrischen Unterschriftsschlüssel anders verhält. Der einzige ernsthafte, mir denkbare Fall, in dem dem Gutmeinenden ein absoluter Schlüsselverlust recht sein kann.

Aber hätten die Shakespeare-Forscher wirklich uneingeschränkten $\mathrm{Spa} ß$ an der Unauffindbarkeit des Schlüssels? Gesetzt den Fall, sie hätten in der Tat Gelegenheit, die Unterschrift zu verifizieren, dann müssen sie sich fragen: War es denn wirklich
Shakespeare, der da unterschrieben hat und nicht irgendein anderer? Hat er gegebenenfalls ausschließlich eigene Werke unterschrieben, oder ist ihm doch etwa ein oder das andere von Christopher Marlowe untergeschlüpft. War der Unterschreibende am Ende doch Edward de Vere, Earl of Oxford, der - wie mancher meint - aus Standesgründen anonym bleiben wollte und "Shakespeare" als Pseudonym benutzte? Keiner von den vielen - oft lächerlichen - Zweifeln an der Verfasserschaft der Dramen, wäre damit ausgeräumt. Es sei denn man wüsste es von zuverlässigen Zeitgenossen, die die Richtigkeit der Verfasserschaft geprüft hatten und beim Unterschreiben wie auch beim Versenken des Unterschriftsschlüssels dabei gewesen waren. Ohne solche Beteiligung Dritter wäre nichts erwiesen. Dann stellt sich aber die Frage nach deren Vertrauenswürdigkeit. Ohne Vertrauen geht nichts. Vertrauen bietet aber keine Sicherheit; es ist eher deren Komplement. Wo in einer Beweiskette ein sicheres Glied fehlt, ersetzt man es durch Vertrauen. Der Nachweis ist dann lediglich so sicher, wie das Vertrauen berechtigt ist.

Das „verlorne slüzzelîn“, liebe Leserinnen und Leser, ging nur die Dame und ihren Geliebten etwas an, ebenso wie die Sorge um den Autoschlüssel nur Harry und mich betraf. Aber bei Shakespeare und seinesgleichen will eine weltweite Kunstgemeinde von vertrauenswürdigen Zeugen wissen, wie sie dran ist; sie sucht gewissermaßen nach „kollektiver“ Erkenntnissicherheit. Wenn sie Zweifel hat und diese ausräumen will, ersetzt sie diese durch neue - wenn auch ihr weniger berechtigt erscheinende Zweifel. Die letzten Zweifel kann man nur mit Vertrauen verdrängen. Endgültige Sicherheit bietet auch nicht ein ,,verlornes slüzzelîn“.

Mit freundlichen Grüßen, Ihr

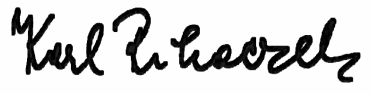

